\title{
Association between Abdominal Obesity and Lumbar Bone Mineral Density According to the Postmenopausal Period in Korean Women
}

\author{
Hyun Young Kim ${ }^{1}$, Sung Soo Kim ${ }^{1,2, *}$, Jong Sung Kim ${ }^{1,2}$, Jin Gyu Jung ${ }^{1,2}$, Seok Joon Yoon ${ }^{1,2}$, Young Hee Jo \\ ${ }^{1}$ Department of Family Medicine, Chungnam National University Hospital, Daejeon; ${ }^{2}$ Department of Family Medicine, Chungnam National University College of \\ Medicine, Daejeon, Korea
}

Background: This study aimed to evaluate the association between abdominal obesity and lumbar bone mineral density (BMD) according to the postmenopausal period of Korean women.

Methods: This was a cross-sectional study. Among the 1,923 women enrolled, 1,138 were included in the study; 785 women were excluded based on conditions that might have affected their bone density. Subjects were divided into short postmenopausal period (SPP, 0-5 years after menopause) and long postmenopausal period (LPP, 6-10 years after menopause). The association between abdominal obesity (waist circumference $\geq 85$ $\mathrm{cm}$ ) and lumbar BMD according to postmenopausal period in Korean women was assessed.

Results: After adjusting for age, exercise amount, alcohol consumption, skeletal muscle mass, and body fat mass, the adjusted odds ratio (OR) for osteoporosis in the group with abdominal obesity compared to the group without abdominal obesity was 0.38 (95\% confidence interval $[\mathrm{Cl}], 0.16-0.88 ; P=0.023$ ) in the SPP group. However, in the LPP and total groups, the adjusted ORs were not significant. The adjusted ORs for low bone mass showed no significance in the SPP, LPP, and total groups.

Conclusion: In the SPP group, the risk of osteoporosis was lower in the group with abdominal obesity than in the group without abdominal obesity. However, in the LPP group, no significant difference was found. Therefore, the effect of abdominal obesity on lumbar BMD may be just for a short period after menopause (0-5 years).
Received June 5, 2017

Reviewed July 3, 2017

Accepted September 12, 2017

*Corresponding author

Sung Soo Kim

(1)

http://orcid.org/0000-0001-9417-7728

Department of Family Medicine, Chungnam National University Hospital, 282 Munhwa-ro, Jung-gu, Daejeon 35015 , Korea

Tel: +82-42-280-8111

Fax: +82-42-280-8373

Email: sungsoo@cnu.ac.kr

Key words: Abdominal obesity, Bone mineral density, Menopause

\section{INTRODUCTION}

Osteoporosis is a common metabolic disorder, with a prevalence of approximately $24 \%$ among Korean women. The risk of osteoporosis increases by more than 7 times after menopause than before menopause. ${ }^{1}$ Postmenopausal osteoporosis is associated with bone loss due to postmenopausal estrogen deficiency. Such bone loss progresses rapidly during 5-10 years after menopause, after which point it begins to slow. ${ }^{2}$

Obesity occurs mostly due to genetic and environmental factors. Estrogen deficiency in postmenopausal women is associated with obesity. As women experience menopause, their risk of developing obesity and metabolic syndrome increases by more than 3 times compared to before menopause, while estrogen therapy can reduce visceral fat tissue. ${ }^{3}$

Considering these points, it is expected that the risk of postmenopausal osteoporosis would increase in obese postmenopausal women. Based on actual clinical trials, the association between body mass index (BMI) and bone mineral density (BMD) is already well-known. However, reported associations between abdominal obesity and bone density in postmenopausal women have varied, with some reporting a positive correlation ${ }^{4-6}$ while others

Copyright (C) 2017 Korean Society for the Study of Obesity

(a) This is an Open Access article distributed under the terms of the Creative Commons Attribution Non-Commercial License (http://creativecommons.org/licenses/by-nc/4.0/) which permits unrestricted non-commercial use, distribution, and reproduction in any medium, provided the original work is properly cited. 
reporting negative correlation ${ }^{7}$ or no association. ${ }^{8,9}$

Most of the studies that examined the association between abdominal obesity and bone density in women considered the menopausal status, but not the postmenopausal period. Since bone loss due to estrogen deficiency progresses rapidly about 5-10 years after menopause, the postmenopausal period is an important factor when assessing bone density. Therefore, we evaluated the association between abdominal obesity and lumbar BMD according to postmenopausal period in Korean women.

\section{METHODS}

\section{Subjects}

Among women who underwent bone density testing at Chungnam National University Hospital Health Promotion Center between March 2014 and July 2016, a total of 1,923 women within 10 years after menopause were identified as candidates for our study. Subsequently, 785 women were excluded based on conditions that might have affected their bone density (16 patients with hyperthyroidism or rheumatoid arthritis; 155 women taking calcium, vitamin $\mathrm{D}$, or drugs for osteoporosis; 31 patients receiving estrogen therapy; 104 patients with restrictions on bone density assessment due to scoliosis, artifact, severe osteoarthritis, soft tissue calcification or spinal operation; 446 women whose menopausal age could not be confirmed due to missing medical records or hysterectomy; 27 current or ex-smokers; and 6 women whose diet included functional foods for menopause). As a result, a final total of 1,138 women were selected as study subjects. This study was approved by the Institutional Review Board of Chungnam National University Hospital (IRB No. 2016-08-010). The informed consent was exempted from the Institutional Review Board because this study is a retrospective and analytical study.

\section{Data collection}

Patient information including medical history, current medications, menopausal age, smoking status, amount of alcohol consumption per week, occupation, and amount of exercise per week was obtained from a self-report questionnaire filled out during health screening.

The study selected women within 10 years after menopause since postmenopausal bone loss progresses rapidly from 5 to 10 years after menopause and slows down thereafter. ${ }^{2}$ The subjects were divided into 2 groups; postmenopausal period of 0 to 5 years (short postmenopausal period, SPP) and postmenopausal period of 6 to 10 years (long postmenopausal period, LPP).

The amount of alcohol consumption was assessed by the frequency of alcohol consumption per week and amount of alcohol consumption per sitting for the past 1 year as reported in the health questionnaire, with $14 \mathrm{~g}$ of alcohol defined as 1 standard drink (hereafter "drink") based on National Institute on Alcohol Abuse and Alcoholism (NIAAA) criteria. ${ }^{10}$ For the amount of exercise per week, those who exercised at least 30 minutes per week were assigned to the exercise group, while those who exercised less than $30 \mathrm{~min}$ or $\mathrm{did}$ not exercise at all were assigned to the non-exercise group.

Height was measured in $\mathrm{cm}$ using an automatic height meter. Weight was measured after at least 12 hours of fasting in $\mathrm{kg}$ with the patient wearing an examination gown. BMI was calculated as weight $(\mathrm{kg})$ divided by height squared $\left(\mathrm{m}^{2}\right)$. A BMI 18.4 or less was defined as underweight; 18.5 to 24.9 as normal; 25 to 29.9 as class 1 obesity; and 30 or more as class 2 obesity. Waist circumference (WC) was measured in $\mathrm{cm}$ at late expiration with a tape measure place above the iliac crest and parallel to the floor. A WC of 84.9 or less $\mathrm{cm}$ was defined as a normal waist and 85 or more $\mathrm{cm}$ was defined as abdominal obesity. The definition of obesity (BMI and WC) was obtained from the Korean Society for the Study of Obesity. ${ }^{11}$ We measured skeletal muscle mass and body fat mass with a Bioimpedance fat analyzer (InBody 770, Biospace Inc., Seoul, Korea).

$\mathrm{BMD}$ was measured in the lumbar spine (1st to 4th lumbar vertebrae) using dual energy X-ray absorptiometry (DXA). A Discovery (Hologic Inc., MA, USA) scanner was used in this study. The lumbar $\mathrm{BMD}$ of 30 patients was measured with two consecutive measurements per patient. The precision error of the lumbar BMD measurement was $1.4 \%$, which was lower than the minimum acceptable precision error of $1.9 \%$ for the lumbar spine. ${ }^{12}$

Since postmenopausal osteoporosis mostly involves the loss of trabecular bone, the femur, which is comprised mostly of cortical bone, was not included.2 Based on their T-scores, the patients were classified as normal BMD ( $\geq-1.0)$, low bone mass $(>-2.5$ and $<$ $-1.0)$ and osteoporosis $(\leq-2.5) .^{12}$ 


\section{Statistical analysis}

The general characteristics of each group were analyzed with $t$ tests and the Chi-square test. To assess the effects of age, menopausal age, height, weight, WC, skeletal muscle mass, body fat mass, amount of exercise per week, and alcohol consumption per week on bone density according to postmenopausal period, oneway ANOVA was performed after dividing the subjects into normal BMD, low bone mass, and osteoporosis groups.

A logistic regression analysis was performed to assess the effects of abdominal obesity on bone density. The odds ratios based on abdominal obesity were calculated after adjusting for age, exercise amount, alcohol consumption, skeletal muscle mass, and body fat mass. The statistics program used was SPSS for Window version 18.0 (SPSS Inc., Chicago, USA), while the significance level was set to $P=0.05$.

\section{RESULTS}

\section{Subject general characteristics}

General characteristics of the subjects are summarized in Table 1.

Table 1. Subject general characteristics

\begin{tabular}{|c|c|c|c|c|c|}
\hline Variables & Subgroup & Total & SPP (0-5 yr) & $\operatorname{LPP}(6-10 \mathrm{yr})$ & $P$ \\
\hline Age (yr) & & $55.4 \pm 3.7(43-66)$ & $53.7 \pm 2.8$ & $58.2 \pm 3.2$ & $<0.001^{*}$ \\
\hline Menopausal age (yr) & & $50.8 \pm 2.8(39-58)$ & $51.2 \pm 2.6$ & $50.3 \pm 3.0$ & $<0.001^{*}$ \\
\hline Weight (kg) & & $58.4 \pm 7.4(39.9-85.5)$ & $58.4 \pm 7.4$ & $58.4 \pm 7.3$ & $0.996^{*}$ \\
\hline \multirow[t]{3}{*}{$\mathrm{BMI}\left[\mathrm{kg} / \mathrm{m}^{2}, \mathrm{n}(\%)\right]$} & Mean & $23.7 \pm 2.8$ & $23.5 \pm 2.8$ & $23.9 \pm 2.8$ & $0.017^{*}$ \\
\hline & $25 \leq \mathrm{BMl}<30$ & $318(27.9 \%)$ & $185(26.4 \%)$ & $133(30.4 \%)$ & \\
\hline & $\mathrm{BMl} \geq 30$ & $27(2.4 \%)$ & $18(2.6 \%)$ & $9(2.1 \%)$ & \\
\hline \multirow[t]{3}{*}{ WC $[\mathrm{cm}, \mathrm{n}(\%)]$} & Mean & $80.0 \pm 8.0$ & $79.3 \pm 8.0$ & $81.1 \pm 7.9$ & $<0.001^{*}$ \\
\hline & $<85$ & $819(72.0 \%)$ & $518(73.9 \%)$ & $301(68.9 \%)$ & \\
\hline & $\geq 85$ & $319(28.0 \%)$ & $183(26.1 \%)$ & $136(31.1 \%)$ & \\
\hline & Self-employment & $152(13.4 \%)$ & $105(15.0 \%)$ & $47(10.8 \%)$ & \\
\hline & Housewife & $607(53.3 \%)$ & $332(47.4 \%)$ & $275(62.9 \%)$ & \\
\hline & Research work & $2(0.2 \%)$ & $2(0.3 \%)$ & $0(0 \%)$ & \\
\hline & The others & $126(11.0 \%)$ & $76(10.8 \%)$ & $50(11.4 \%)$ & \\
\hline \multirow[t]{3}{*}{ Alcohol consumption } & Non-drinkers & $773(67.9 \%)$ & 451 (64.3\%) & $322(73.7 \%)$ & \\
\hline & Drinkers & $365(32.1 \%)$ & $250(35.7 \%)$ & $115(26.3 \%)$ & \\
\hline & Alcohol amount (g/week) & $7.4 \pm 23.4$ & $8.4 \pm 24.1$ & $5.9 \pm 22.0$ & $0.081^{*}$ \\
\hline \multirow[t]{3}{*}{ Exercise } & None & $593(52.1 \%)$ & $362(51.6 \%)$ & $231(52.9 \%)$ & \\
\hline & Regular & $545(47.9 \%)$ & $339(48.4 \%)$ & $206(47.1 \%)$ & \\
\hline & Exercise amount (hours/week) & $1.7 \pm 2.5$ & $1.8 \pm 2.6$ & $1.6 \pm 2.4$ & $0.373^{*}$ \\
\hline
\end{tabular}

Values are expressed as mean \pm standard deviation (SD) or number (min. to max. or \%).

${ }^{*}$ Obtained by $t$-test; ${ }^{\dagger}$ Obtained by Chi-square test for categorical variables in BMD.

SPP, short postmenopausal period (0-5 years after menopause); LPP, long postmenopausal period (6-10 years after menopause); BMl, body mass index; WC, waist circumference; Regular exercise, more than 30 min per week; BMD, bone mineral density (L1 to L4); Normal, T-score $\geq-1.0$; Low bone mass, $-2.5<$ T-score $<-1.0$; Osteoporosis, T-score $\leq-2.5$. 
Table 2. Factors related to BMD in women with a short postmenopausal period (0-5yr)

\begin{tabular}{|c|c|c|c|c|}
\hline & Normal $(n=219)$ & Low bone mass $(n=365)$ & Osteoporosis $(\mathrm{n}=117)$ & $P^{*}$ \\
\hline Age (yr) & $53.5 \pm 2.6$ & $53.7 \pm 2.9$ & $54.1 \pm 3.2$ & 0.185 \\
\hline Menopause age (yr) & $51.4 \pm 2.5$ & $51.0 \pm 2.6$ & $51.2 \pm 2.8$ & 0.351 \\
\hline Height (cm) & $158.2 \pm 4.8^{\mathrm{a}}$ & $157.5 \pm 4.6^{\mathrm{a}, \mathrm{b}}$ & $156.5 \pm 4.0^{b}$ & 0.008 \\
\hline Weight (kg) & $60.9 \pm 7.4^{\mathrm{a}}$ & $58.0 \pm 6.9^{b}$ & $55.0 \pm 7.0^{c}$ & $<0.001$ \\
\hline $\mathrm{BMl}\left(\mathrm{kg} / \mathrm{m}^{2}\right)$ & $24.3 \pm 2.8^{\mathrm{a}}$ & $23.4 \pm 2.6^{b}$ & $22.5 \pm 2.7^{c}$ & $<0.001$ \\
\hline $\mathrm{WC}(\mathrm{cm})$ & $81.6 \pm 7.7^{\mathrm{a}}$ & $78.8 \pm 7.8^{b}$ & $76.7 \pm 8.3^{c}$ & $<0.001$ \\
\hline Skeletal muscle mass (kg) & $23.1 \pm 5.2^{\mathrm{a}}$ & $21.6 \pm 4.0^{b}$ & $21.3 \pm 4.7^{b}$ & $<0.001$ \\
\hline Body fat mass (kg) & $20.5 \pm 5.0^{\mathrm{a}}$ & $19.0 \pm 4.9^{b}$ & $17.1 \pm 4.8^{c}$ & $<0.001$ \\
\hline Exercise amount (hour/week) & $1.6 \pm 2.3$ & $1.9 \pm 2.9$ & $1.7 \pm 2.2$ & 0.311 \\
\hline Alcohol consumption (g/week) & $9.3 \pm 21.2$ & $9.3 \pm 28.5$ & $3.8 \pm 9.5$ & 0.079 \\
\hline
\end{tabular}

Values are expressed as mean \pm standard deviation (SD).

${ }^{*}$ Obtained by one-way ANOVA test, the same letters indicate non-significant differences between groups based on Tukey's multiple comparison test.

BMD, bone mineral density; Short postmenopausal period, 0-5 years after menopause; Normal, T-score $\geq-1.0 ;$ Low bone mass, $-2.5<$ T-score $<-1.0$; Osteoporosis, T-score $\leq-2.5$; BMl, body mass index; WC, waist circumference.

Weight, skeletal muscle mass, body fat mass, alcohol consumption, and exercise amount were not significantly different between the SPP and LPP groups. Menopausal age and height were significantly higher in the SPP than in the LPP. WC and BMI were significantly higher in the LPP than in the SPP.

Lumbar BMD was significantly higher in the SPP group than in the LPP group $(0.893 \pm 0.115,0.845 \pm 0.114, P<0.001)$. There was a significant difference between the SPP and the LPP groups in terms of the proportion of normal, low bone mass, and osteoporosis groups $(P<0.001)$. The proportions of normal, low bone mass, and osteoporosis groups were $31.2 \%, 52.1 \%$, and $16.7 \%$ in the SPP group, versus $17.8 \%, 51.3 \%$, and $30.9 \%$ in the LPP group, respectively.

\section{Factors related to BMD in women with a short postmenopausal period ( 0 to 5 years)}

Table 2 shows the factors related to BMD in women with a postmenopausal period of 0 to 5 years. Height was significantly less in the osteoporosis group than in the normal BMD group $(P=0.008)$. Skeletal muscle mass was significantly higher in the normal BMD group than in the low bone mass and osteoporosis groups $(P<$ 0.001). Weight, BMI, WC, and body fat mass were significantly the highest to lowest in the normal, low bone mass, and osteoporosis groups $(P<0.001)$. Age, menopausal age, exercise amount, and alcohol consumption were not significantly different among the three groups.

\section{Factors related to $\mathrm{BMD}$ in women with a long postmenopausal period ( 6 to 10 years)}

Table 3 shows factors related to BMD in women with a postmenopausal period of 6 to 10 years. Weight, BMI, WC, and skeletal muscle mass were significantly the highest to lowest in the normal, low bone mass, and osteoporosis groups $(P<0.001)$. Body fat mass was significantly higher in the normal and low bone mass groups than in the osteoporosis groups $(P<0.001)$. Age, menopausal age, height, exercise amount, and alcohol consumption were not significantly different among the three groups.

\section{Odds ratios for low bone mass and osteoporosis according to abdominal obesity}

After adjusting for age, exercise amount, alcohol consumption, skeletal muscle mass, and body fat mass, the adjusted odds ratio (OR) for low bone mass in the group with abdominal obesity in comparison with the group without abdominal obesity in the SPP group was 0.76 (95\% confidence interval [CI], 0.45-1.29; $P=$ 0.314 ), 1.20 (95\% CI, 0.56-2.54; $P=0.642$ ) in the LPP group and 0.89 (95\% CI, 0.58-1.36; $P=0.592$ ) in the total group, which were not significant. In addition, after adjusting for age, exercise amount, alcohol consumption, skeletal muscle mass, and body fat mass, the adjusted OR for osteoporosis in the group with abdominal obesity in comparison with the group without abdominal obesity in the SPP group was 0.38 (95\% CI, 0.16-0.88; $P=0.023$ ), which was significant. However, in the LPP and total groups, the ORs were 1.33 
Table 3. Factors related to BMD in women with a long postmenopausal period (6-10 yr)

\begin{tabular}{|c|c|c|c|c|}
\hline & Normal $(n=78)$ & Low bone mass $(n=224)$ & Osteoporosis $(n=135)$ & $P^{*}$ \\
\hline Age (yr) & $58.2 \pm 3.3$ & $58.1 \pm 3.3$ & $58.3 \pm 3.2$ & 0.881 \\
\hline Menopausal age (yr) & $50.5 \pm 3.0$ & $50.3 \pm 3.1$ & $50.2 \pm 3.0$ & 0.795 \\
\hline Height (cm) & $157.3 \pm 5.4$ & $156.2 \pm 5.1$ & $155.6 \pm 4.8$ & 0.054 \\
\hline Weight (kg) & $62.2 \pm 7.7^{\mathrm{a}}$ & $58.5 \pm 7.0^{b}$ & $56.0 \pm 6.8^{c}$ & $<0.001$ \\
\hline $\mathrm{BMI}\left(\mathrm{kg} / \mathrm{m}^{2}\right)$ & $25.1 \pm 2.8^{\mathrm{a}}$ & $24.0 \pm 2.8^{b}$ & $23.2 \pm 2.7^{c}$ & $<0.001$ \\
\hline$W C(\mathrm{~cm})$ & $83.8 \pm 7.5^{\mathrm{a}}$ & $81.3 \pm 7.9^{b}$ & $79.0 \pm 7.7^{c}$ & $<0.001$ \\
\hline Skeletal muscle mass $(\mathrm{kg})$ & $23.6 \pm 5.8^{\mathrm{a}}$ & $21.9 \pm 4.6^{b}$ & $20.4 \pm 2.5^{c}$ & $<0.001$ \\
\hline Body fat mass (kg) & $21.2 \pm 4.7^{\mathrm{a}}$ & $20.0 \pm 4.9^{\mathrm{a}}$ & $18.3 \pm 4.7^{b}$ & $<0.001$ \\
\hline Exercise amount (hour/week) & $1.7 \pm 2.2$ & $1.8 \pm 2.7$ & $1.3 \pm 1.7$ & 0.101 \\
\hline Alcohol consumption (g/week) & $6.2 \pm 18.4$ & $6.0 \pm 23.3$ & $5.7 \pm 21.7$ & 0.982 \\
\hline
\end{tabular}

Values are expressed as mean \pm standard deviation (SD).

*Obtained using the one-way ANOVA test, the same letters indicate non-significant differences between groups based on Tukey's multiple comparison test.

BMD, bone mineral density; Long postmenopausal period, 6-10 years after menopause; Normal, T-score $\geq-1.0$; Low bone mass, -2.5 $<$ T-score $<-1.0$; 0steoporosis, T-score $\leq-2.5$; $\mathrm{BMI}$, body mass index; WC, waist circumference.

Table 4. Odds ratios for low bone mass and osteoporosis according to abdominal obesity

\begin{tabular}{|c|c|c|c|c|c|c|}
\hline \multirow{2}{*}{ Postmenopausal period (yr) } & \multirow{2}{*}{ WC (cm) } & \multirow{2}{*}{$N$} & \multicolumn{2}{|c|}{ Low bone mass } & \multicolumn{2}{|c|}{ Osteoporosis } \\
\hline & & & $\mathrm{OR}^{*}$ & $P^{\dagger}$ & $\mathrm{OR}^{*}$ & $P^{\dagger}$ \\
\hline \multirow[t]{2}{*}{ SPP (0 to 5) } & $<85$ & 518 & 1 & & 1 & \\
\hline & $\geq 85$ & 183 & $0.76(0.45-1.29)$ & 0.314 & 0.38 (0.16-0.88) & 0.023 \\
\hline \multirow[t]{2}{*}{$\operatorname{LPP}(6$ to 10$)$} & $<85$ & 301 & 1 & & 1 & \\
\hline & $\geq 85$ & 136 & 1.20 (0.56-2.54) & 0.642 & 1.33 (0.52-3.37) & 0.551 \\
\hline \multirow[t]{2}{*}{ Total (0 to 10) } & $<85$ & 819 & 1 & & 1 & \\
\hline & $\geq 85$ & 319 & 0.89 (0.58-1.36) & 0.592 & $0.66(0.36-1.22)$ & 0.186 \\
\hline
\end{tabular}

All values are adjusted by age, amount of exercise per week, alcohol consumption per week, skeletal muscle mass, and body fat mass with a $95 \%$ confidence interval.

${ }^{*}$ Odds ratios were obtained using multiple logistic regression analysis; ${ }^{\dagger}$ Obtained by logistic regression analysis.

SPP, short postmenopausal period (0-5 years after menopause); LPP, long postmenopausal period (6-10 years after menopause); WC, waist circumference; WC $\geq 85 \mathrm{~cm}$, abdominal obesity.

(95\% CI, 0.52-3.37; $P=0.551)$ and 0.66 (95\% CI, 0.36-1.22; $P=$ 0.186 ), respectively, which were not significant (Table 4).

\section{DISCUSSION}

The purpose of this study was to evaluate the association between abdominal obesity and lumbar BMD according to postmenopausal period in Korean women. The association between abdominal obesity and BMD is controversial.

In this study, patients in the LPP group (6 to 10 years after menopause) showed a lower BMD and a higher proportion of osteoporosis than those in the SPP group ( 0 to 5 years after menopause) (Table 1). This result correlates with a previous study that showed decreased BMD and increased incidence of osteoporosis as age increased occurred in postmenopausal women. ${ }^{13}$
Weight, BMI, WC, skeletal muscle mass, and body fat mass in the osteoporotic group were significantly lower than in the normal BMD group regardless of short and long postmenopausal periods (Tables 2 and 3). These results were similar to study results that showed $\mathrm{BMD}$ was positively associated with $\mathrm{WC}$, and that $\mathrm{WC}$ in osteoporotic women was significantly lower than in women without osteoporosis. ${ }^{46}$

The BMI of Korean men is the highest when they are in their thirties to fifties and decreases when they are in their sixties, whereas the BMIs of Korean women increase continuously until they are in their sixties. The WC of men remain consistent from when they are in their thirties to when they are in their seventies, whereas those of women increase gradually with age and increase rapidly after their fifties. ${ }^{14}$ This study similarly found that the LPP group with a higher age had a significantly higher BMI and WC than the SPP 
group (Table 1). The main cause of the rapid increase in WC after the fifties may be the effect of postmenopausal estrogen deficiency. ${ }^{3}$

Low BMI is an important risk factor for low bone mass and increased bone loss in early postmenopausal women. ${ }^{15}$ Our study also showed that BMI and WC were significantly the highest to lowest for normal, low bone mass, and osteoporosis in both the SPP and LPP groups (Tables 2 and 3 ).

In this study, there was no relationship found between BMD and WC in the total group (Table 4). Dytfeld et al. ${ }^{4}$ showed that there was a weak positive correlation between WC and lumbar and femur BMD in postmenopausal women $(r=0.25$ and $r=0.36$ respectively). In the study by Dytfeld et $\mathrm{al}^{4}{ }^{4}$, the average age of the subjects was 69.5 years, which was more than 10 years older than the average age of the subjects in our study, which was 55.4 years. Such differences in study design may explain the discrepancy between the results of our study and others.

However, other studies have demonstrated that waist to hip ratio was negatively associated with lumbar BMD and waist to hip ratio of osteoporotic women was higher than that of normal women. ${ }^{7}$ Because this study did not confirm menopausal status and used waist to hip ratios instead of WC, this result cannot be applied to the association between $\mathrm{WC}$ and $\mathrm{BMD}$. Other studies have suggested that WC was not associated with $\mathrm{BMD} .{ }^{8,9}$ One study used femoral neck BMD instead of lumbar BMD in postmenopausal women. ${ }^{8}$ Because postmenopausal osteoporosis involves mainly trabecular bone, lumbar BMD is more suitable for BMD evaluation than femur BMD in women with rapid bone loss observed approximately 5 to 10 years after menopause. ${ }^{2}$ A study examining the association between metabolic syndrome and BMD showed a weak negative association $(\mathrm{r}=-0.20)$ between $\mathrm{WC}$ and lumbar BMD in premenopausal women, but not in postmenopausal women. ${ }^{9}$ The average age of the subjects in this study was 61.1 years, which was five years older than that of the subjects of our study. In addition, the correlation between WC and lumbar BMD in postmenopausal women was not significant, as with the outcome of this study. This outcome appears to be related to the small target group of older subjects (mean age, 61.1 years).

The postmenopausal period and region of interest for BMD (lumbar or femur) may explain the different results between WC and $\mathrm{BMD}$ in postmenopausal women. Trabecular bone loss in verte- brae increases more rapidly than cortical bone loss in long bones during the first 8 to 10 years after menopause. Additionally, loss of multiple estrogen-induced restraining actions on osteoclast bone resorption leads to upregulation of rapid bone loss during this period. ${ }^{16}$ Therefore, consideration of the postmenopausal period and BMD region of interest are important when evaluating the association between WC and BMD. We considered the postmenopausal period when evaluating the association between WC and lumbar BMD.

In this study, with respect to the SPP group, the group with abdominal obesity showed a significantly lower OR for osteoporosis than the group without abdominal obesity (Table 4). In postmenopausal women, while the secretion of estrogen from ovaries ceases, estrogen concentration is somewhat maintained by the peripheral conversion of androstenedione to estrone. The degree to which androstenedione is converted into estrone increases as body weight increases. ${ }^{17}$ Therefore, the reason for the lower risk of osteoporosis in the SPP abdominal obesity group compared to the group without abdominal obesity is estimated to be related to the peripheral conversion of androstenedione to estrone. This study is cross-sectional and causality cannot be established because no evaluation was performed with respect to estrogen. Considering that the OR for osteoporosis showed no significance in the LPP group, the effect of abdominal obesity can be estimated to last for a short period (0-5 years after menopause). In the SPP group, the OR for low bone mass was 0.76 , which was not significant. Therefore, the effect of abdominal obesity described herein appears to be more frequent in osteoporosis than in low bone mass.

The odds ratio or relative risk may be more relevant to clinical practice than the effect of WC on BMD in postmenopausal women. The effect of abdominal obesity (increased WC) on BMD was evaluated by odds ratios for low bone mass and osteoporosis instead of their association, unlike previous studies. In addition, the postmenopausal period was considered in this study.

There were some limitations to our study. First, we could not establish causality between abdominal obesity and BMD due to the limitations of the study's cross-sectional design. Second, subjects were not representative of the Korean population since it only included patients from a regional university hospital. Third, we did not consider family history of fracture, which can affect BMD. Fourth, spinal fracture was not evaluated using a thoracolumbar 
spine X-ray but with lumbar DXA.

Finally, in the SPP group (0-5 years after menopause), the risk of osteoporosis was lower in the group with abdominal obesity than in the group without abdominal obesity. However, in the LPP group (6-10 years after menopause), no significant difference was found. Therefore, the effect of abdominal obesity on lumbar BMD may be for a short period only (0-5 years after menopause).

\section{CONFLICTS OF INTEREST}

The authors declare no conflict of interest.

\section{REFERENCES}

1. Shin CS, Choi HJ, Kim MJ, Kim JT, Yu SH, Koo BK, et al. Prevalence and risk factors of osteoporosis in Korea: a community-based cohort study with lumbar spine and hip bone mineral density. Bone 2010;47:378-87.

2. Pietschmann P, Rauner M, Sipos W, Kerschan-Schindl K. Osteoporosis: an age-related and gender-specific disease-a minireview. Gerontology 2009;55:3-12.

3. Lizcano F, Guzmán G. Estrogen deficiency and the origin of obesity during menopause. Biomed Res Int 2014;2014:757461.

4. Dytfeld J, Ignaszak-Szczepaniak M, Gowin E, Michalak M, Horst-Sikorska W. Influence of lean and fat mass on bone mineral density (BMD) in postmenopausal women with osteoporosis. Arch Gerontol Geriatr 2011;53:e237-42.

5. Fodor D, Vesa S, Albu A, Simon S, Craciun A, Muntean L. The relationship between the metabolic syndrome and its components and bone status in postmenopausal women. Acta Physiol Hung 2014;101:216-27.

6. Abbasi M, Farzam SA, Mamaghani Z, Yazdi Z. Relationship between metabolic syndrome and its components with bone densitometry in postmenopausal women. Diabetes Metab Syndr 2016 Dec 15 [Epub]. https://doi.org/10.1016/j.dsx. 2016.12.008

7. Kim CJ, Oh KW, Rhee EJ, Kim KH, Jo SK, Jung CH, et al. Relationship between body composition and bone mineral density (BMD) in perimenopausal Korean women. Clin En- docrinol (Oxf) 2009;71:18-26.

8. Kim HY, Choe JW, Kim HK, Bae SJ, Kim BJ, Lee SH, et al. Negative association between metabolic syndrome and bone mineral density in Koreans, especially in men. Calcif Tissue Int 2010;86:350-8.

9. Yoo HJ, Park MS, Yang SJ, Kim TN, Lim KI, Kang HJ, et al. The differential relationship between fat mass and bone mineral density by gender and menopausal status. J Bone Miner Metab 2012;30:47-53.

10. Willenbring ML, Massey SH, Gardner MB. Helping patients who drink too much: an evidence-based guide for primary care clinicians. Am Fam Physician 2009;80:44-50.

11. Kim MK, Lee WY, Kang JH, Kang JH, Kim BT, Kim SM, et al. 2014 clinical practice guidelines for overweight and obesity in Korea. Korean J Obes 2014;23:217-21.

12.2015 ISCD Official Positions - Adult. The International Society For Clinical Densitometry. Supersedes all prior "Official Positions" publications. [accessed 2015 June 16]; Available from: URL: http://www.iscd.org/official-positions/2015-iscdofficial-positions-adult

13. Park EJ, Joo IW, Jang MJ, Kim YT, Oh K, Oh HJ. Prevalence of osteoporosis in the Korean population based on Korea National Health and Nutrition Examination Survey (KNHANES), 2008-2011. Yonsei Med J 2014;55:1049-57.

14. Ministry of Health and Welfare, Korea Centers for Disease Control and Prevention. Korea Health Statistics 2013: Korea National Health and Nutrition Examination Survey (KNHANES VI-1); 2014. p. 50-1.

15. Ravn P, Cizza G, Bjarnason NH, Thompson D, Daley M, Wasnich $\mathrm{RD}$, et al. Low body mass index is an important risk factor for low bone mass and increased bone loss in early postmenopausal women. J Bone Miner Res 1999;14:1622-7.

16. Clarke BL, Khosla S. Physiology of bone loss. Radiol Clin North Am 2010;48:483-95.

17. MacDonald PC, Edman CD, Hemsell DL, Porter JC, Siiteri PK. Effect of obesity on conversion of plasma androstenedione to estrone in postmenopausal women with and without endometrial cancer. Am J Obstet Gynecol 1978;130:448-55. 